\title{
Surgical treatment of recurrent gastric cancer
}

\author{
Álvaro Díaz de Liaño ${ }^{1}$, Concepción Yarnoz ${ }^{1}$, Rubén Aguilar ${ }^{2}$, Cristina Artieda $^{1}$, and Héctor Ortiz ${ }^{1}$ \\ ${ }^{1}$ Esophagogastric Unit, Department of General and Gastrointestinal Surgery, Hospital Virgen del Camino, C/ Pintor Maeztu 2, $8^{\circ} \mathrm{C}$, \\ Pamplona 31008, Navarra, Spain \\ ${ }^{2}$ Department of General Surgery, Hospital Central Instituto de Previsión Social, Asunción, Paraguay
}

\begin{abstract}
Background. The value of programs to detect recurrence of a previously operated gastric cancer is a controversial subject. D2 lymphadenectomy achieves better local control and a lower local recurrence rate than D1 lymphadenectomy.

Methods. The results achieved with surgical treatment of recurrent gastric cancer in a series of 126 consecutive patients who had been treated with gastrectomy with D2 lymphadenectomy are reported. In-hospital mortality was $1.6 \%$.

Results. Follow-up of the remaining 124 patients for a median period of 73.6 months detected recurrence in 46 patients (37\%). There were 14 local, 12 peritoneal, and 15 distant recurrences. Recurrent gastric cancer was detected within 2 years in $76 \%$ of the patients. Of the 46 patients with recurrence, 5 patients $(11 \%)$ were selected for surgery (4 local and 1 peritoneal recurrence). The median disease-free time after surgery for recurrence was 26 months (range, 14-58 months). There was no in-hospital mortality. The median survival time after surgery for recurrence was 26 months (range, 9-87 months). One patient died during follow-up, while none of the other patients have any evidence of new recurrence.

Conclusion. Surgery for recurrent gastric cancer is a valid alternative in selected patients, provided it is performed by specialized work teams.
\end{abstract}

Key words Recurrence · Gastric cancer · Surgery

\section{Background}

The value of programs to detect recurrent gastric cancer is a controversial subject because of the great difficulty of its diagnosis, the high cost involved in follow-up programs, and the fact that very few studies have shown the benefits of treating this group of patients [1, 2].

Offprint requests to: Á. Díaz de Liaño

Received: July 17, 2007 / Accepted: November 19, 2007
Moreover, recurrence in more than one location may be diagnosed simultaneously, which usually makes the condition not amenable to surgery $[2,3]$. The prognosis of patients with recurrent gastric cancer is usually considered to be poor because of several factors, including a low resectability rate and a poor tolerance of treatment, with increased morbidity and mortality $[1,4]$.

The incidence of the various types of recurrence varies depending on the reported studies. D2 Lymphadenectomy achieves, according to most publications that advocate it, improved local control of the disease and a decrease in the local recurrence rate [4-11]. However, the stage of disease at the time of the initial surgery influences peritoneal and hematogenous recurrence $[5,11]$. The most common hematogenous recurrences occur in the liver, and their surgical treatment is not widely accepted, but studies about the treatment of liver metastases from gastric cancer have reported improved survival in such surgically treated patients [11-16]. Another type of peritoneal recurrence, Krukenberg tumor (ovarian metastasis), has a low incidence rate, ranging from $0.7 \%$ to $6.7 \%$. Its resection is recommended by some authors, who found evidence of increased survival in patients who underwent surgery [17-19].

We report the results achieved with surgical treatment for recurrent gastric cancer detected in a series of 126 consecutive patients who had undergone prior gastrectomy with D2 lymphadenectomy at the Department of General and Gastrointestinal Surgery (Esophagogastric Unit) of Hospital Virgen del Camino, Pamplona, Navarra, Spain.

\section{Patients and methods}

A series of 126 consecutive patients diagnosed with gastric adenocarcinoma with no distant metastasis who underwent D2 gastrectomy according to the recommen- 
Table 1. Characteristics of the 126 consecutive patients (D2 gastrectomy)

\begin{tabular}{|c|c|}
\hline \multirow{2}{*}{\multicolumn{2}{|c|}{$\begin{array}{l}\text { Median age, in years (range) } \\
\text { Sex }(\%)\end{array}$}} \\
\hline & \\
\hline Male & $89(70.6)$ \\
\hline \multicolumn{2}{|l|}{$\operatorname{ASA}(\%)^{\mathrm{a}}$} \\
\hline $\mathrm{I}$ & $10(7.9)$ \\
\hline II & $35(27.8)$ \\
\hline III & $78(61.9)$ \\
\hline IV & $3(2.4)$ \\
\hline \multicolumn{2}{|l|}{ Tumor location (\%) } \\
\hline Upper third & $36(28.6)$ \\
\hline Middle third & $35(27.8)$ \\
\hline Lower third & $51(40.5)$ \\
\hline Gastric stump & $4(3.2)$ \\
\hline \multicolumn{2}{|l|}{ Lauren type (\%) } \\
\hline Intestinal & 77 (61.1) \\
\hline Diffuse & $42(33.3)$ \\
\hline Indeterminate & $7(5.6)$ \\
\hline \multicolumn{2}{|l|}{ Type of gastrectomy (\%) } \\
\hline Total & $95(75.4)$ \\
\hline Subtotal & $31(24.6)$ \\
\hline Median tumor size, in cm (range) & $5(1-16)$ \\
\hline \multicolumn{2}{|l|}{ Associated surgical procedures (\%) } \\
\hline Splenectomy & $22(17.5)$ \\
\hline Left pancreatectomy & $9(7.1)$ \\
\hline Colectomy & $4(3.2)$ \\
\hline Hepatectomy & $4(3.2)$ \\
\hline Nephrectomy & $1(0.8)$ \\
\hline \multicolumn{2}{|l|}{ Infiltration of surgical margins (\%) } \\
\hline Upper & $6(4.8)$ \\
\hline Lower & $5(4.0)$ \\
\hline \multicolumn{2}{|l|}{ Type of surgery (\%) } \\
\hline R0 & $99(78.6)$ \\
\hline $\mathrm{R} 1$ & $27(21.4)$ \\
\hline \multicolumn{2}{|l|}{ Infiltration of other organs (\%) } \\
\hline Pancreas & $3(2.4)$ \\
\hline Colon & $4(3.2)$ \\
\hline Liver & $1(0.8)$ \\
\hline Spleen & $1(0.8)$ \\
\hline Esophageal hiatus & $1(0.8)$ \\
\hline Kidney & $1(0.8)$ \\
\hline Median no. of lymph nodes excised per patient & 32.5 \\
\hline \multicolumn{2}{|l|}{$\mathrm{N}$ stage $(\%)$} \\
\hline $\mathrm{NO} \mathrm{b}^{\mathrm{b}}$ & $51(40.5)$ \\
\hline $\mathrm{N} 1^{\mathrm{c}}$ & $42(33.3)$ \\
\hline $\mathrm{N} 2^{\mathrm{d}}$ & $17(13.5)$ \\
\hline $\mathrm{N} 3^{\mathrm{e}}$ & $16(12.7)$ \\
\hline \multicolumn{2}{|l|}{ Staging $(\%)$} \\
\hline 0 & $6(4.8)$ \\
\hline IA & $22(17.5)$ \\
\hline IB & $28(22.2)$ \\
\hline II & $13(10.3)$ \\
\hline IIIA & $21(16.7)$ \\
\hline IIIB & $12(9.5)$ \\
\hline IV & $24(19.0)$ \\
\hline
\end{tabular}

${ }^{a}$ American Society of Anesthesiology classification of physical status

${ }^{\mathrm{b}}$ No positive lymph nodes

${ }^{\mathrm{c}} 1$ to 6 positive lymph nodes

d 7 to 15 positive lymph nodes

${ }^{\mathrm{e}}$ More than 15 positive lymph nodes dations of the Japanese Research Society for Gastric Cancer were prospectively studied (from April 1998 to March 2006). Staging was performed according to the TNM classification included in the 5th edition of the International Union Against Cancer (UICC) and the American Joint Committee on Cancer (AJCC) of 1997 [20]. Table 1 shows the general characteristics of the patient group.

In-hospital mortality was $1.6 \%$ and morbidity, $29.4 \%$. The remaining 124 patients (both $\mathrm{R} 0$ and $\mathrm{R} 1$ ) were followed up. Follow-up included clinical, laboratory, and ultrasonographic monitoring in all patients every 6 months for the first 2 years after surgery and every year thereafter. In addition, when recurrence was suspected, endoscopy with biopsy, computed tomography (CT), and X-ray of the gastrointestinal tract with contrast were performed as appropriate for each individual patient. Laboratory tests included, among other measurements, carcinoembryonic antigen (CEA) and carbohydrate antigen (CA) 19-9 levels. Median follow-up time was 73.6 months.

Recurrences were grouped as locoregional, peritoneal, and distant. Locoregional recurrences included those occurring in the gastric bed or the gastric remnant of anastomosis, and retroperitoneal lymphadenopathies of the upper abdomen. Peritoneal recurrences included those involving the ovaries (Krukenberg tumors), carcinomatosis, and positive cytology. Distant recurrences were those occurring in other organs and in nonregional lymph nodes. The disease-free period was defined as the time, in months, from the surgery for gastric cancer to the diagnosis of recurrence.

\section{Results}

Recurrence was found in 46 patients (37\%), of whom 15 patients had distant recurrence; 14, local recurrence; and 12, peritoneal recurrence. Among the 5 patients who had recurrence in more than one location, 3 patients had both distant and local recurrence and 2 patients had both local and peritoneal recurrence.

In the first 2 years, recurrence was found in 35 patients $(76 \%)$. Of these, 11 patients experienced local recurrence; 10 , peritoneal recurrence; and 14, distant recurrence.

Among the 46 patients with recurrence, 5 patients $(11 \%)$ who could benefit from surgery, based on the data from clinical assessments and supplemental tests performed, were selected for tumor resection, and their findings are analyzed in this article.

Treatment in the remainder of the patients with recurrence, whose recurrences were not resected (41 patients), varied; for instance, of the 10 patients with local recurrence, 2 received chemotherapy, 2 underwent 
surgery (in 1 patient a jejunal bypass was carried out and in the other patient there was no surgical solution), 2 patients received minimally invasive treatment techniques (a percutaneous biliary drainage and an endoscopic prosthesis), and 4 patients did not receive any type of treatment. For the 11 patients with peritoneal recurrence, surgical intervention was performed in 5 patients ( 2 patients had a bypass performed and in 3 patients we were only able to confirm the disease); 1 patient received chemoradiation, and 5 patients did not receive any type of treatment. Only 6 of the 15 patients with distant recurrence underwent chemotherapy. One of the 3 patients with distant and local recurrence received chemotherapy. An ileostomy was performed in 1 patient with local and peritoneal recurrence and chemotherapy was given to the other such patient.

Table 2 lists the characteristics of the patients in the study who received resection for recurrence. As previously noted, all patients had undergone a D2 lymphadenectomy. Patients 1,2, and 5 were asymptomatic at the time recurrence was diagnosed. In patient 1 , recurrence was suspected because of an increased CEA level with other laboratory test results being normal, except for a raised alkaline phosphatase level. A CT scan was done, and showed a mass in the small bowel. At the surgical intervention, a recurrence was found in the Roux-en-Y jejunal limb $12 \mathrm{~cm}$ below the esophagojejunal anastomosis. For this reason the recurrence was considered to be locoregional. Histological study indicated a jejunal metastasis from adenocarcinoma with the same features as the primary tumor. In patient 2, biopsies of the anastomotic mouth ulcer were positive for adenocarcinoma, while in patient 5 a tumor mass was seen in the pelvis in a control CT scan. None of these three patients received adjuvant treatment following the surgery for recurrence resection. In patients 3 and 4, dysphagia was the common symptom allowing for diagnosis of recurrence. Patients 1 to 4 were considered to have locoregional recurrence, while the remaining recurrence was considered to be peritoneal in nature (Krukenberg tumor).

Table 2 shows both the recurrence-free interval from the curative resection to recurrence and survival after the recurrence resection. The median disease-free time after the surgery for recurrence was 26 months (range, 14-58 months), and median survival time was 26 months (range, 9-87 months). At the time of writing this article, only 1 patient (patient 5) had died, and none of the 4 surviving patients showed evidence of new recurrence. Of the 41 patients with recurrences that were not resected, all but 3 had died; the median survival was 2.4 months (range, 0.17-30.47 months).

\section{Discussion}

Surgical treatment is recommended by some authors for local recurrence of gastric cancer, but the number of patients with locally recurrent gastric cancer in whom surgery may be attempted, based on oncological criteria, is low [11, 16]. Bohner et al. [1] reported that they were able to treat $21 \%$ of their patients by surgery based on oncological criteria, but when the group of patients who underwent surgery alone (i.e., without prior oncological therapy) was considered, the proportion they were able to treat fell to less than $12 \%$. In the group studied by Yoo et al. [5], the proportion of patients treated with curative surgery was low, at 3.7\%. Shchepotin et al. [21] reported a 53\% resection rate for local recurrence, but almost $60 \%$ of their patients received some type of oncological therapy before surgery. In our study, oncological surgical treatment was achieved in $11 \%$ of patients, a proportion similar to that reported by Bohner et al. [1]. None of our five patients had received any type of oncological therapy.

In our patient 2 , the only patient who had previously undergone a subtotal gastrectomy for a tumor with an intestinal histological type, recurrence occurred at the gastric remnant level, and surgery consisted of the resection of such remnant. The gastric remnant has been reported by several authors to be one of the most common sites of local recurrence. Some authors, including Lehnert et al. [11], think that this type of recurrence occurs mainly in gastric cancer of diffuse type, because of its discontinuous growth pattern, because tumor cells may remain in the gastric remnant even in patients with tumor-free resection margins $[1,5,21]$. Recurrence at the gastric remnant level may also appear in patients treated for an early gastric cancer [22].

Marrelli et al. [10], as well as other authors [9], reported that a greater number of local recurrences were found during the follow-up of tumors of an intestinal histological type. Our results agree with this finding, as $100 \%$ of our patients with local recurrence had an intestinal histological type.

Our findings are similar to those obtained and reported by D'Angelica et al. [4], who found a predominance of males with tumors of the T1 and T2 intestinal histological types to be more susceptible to experiencing local and distant recurrence. Three of our four patients with regional recurrence were males, and three patients had T1/T2 tumors. By contrast, our data do not agree with the local recurrence predictors reported by Schwartz et al. [3], according to which the chance of recurrence in T1/T2 tumors is only $13 \%$. None of our patients had N2 or N3 nodal involvement, which is considered by the model of Marrelli et al. [23] as a recognized marker of the patient groups at risk of recurrence. 
Á. Díaz de Liaño et al.: Resection of gastric cancer recurrence

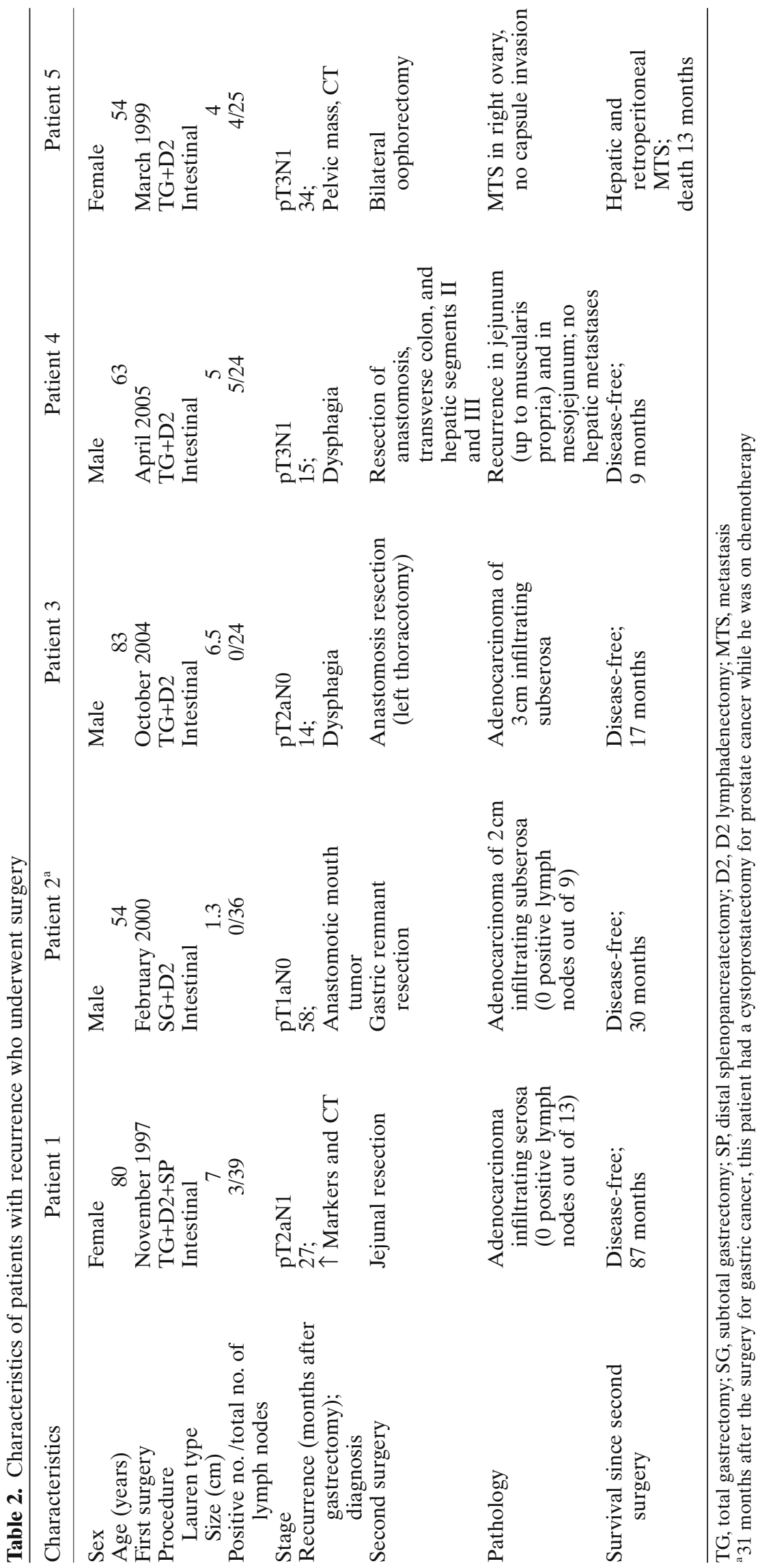


The characteristics of our single patient with resected peritoneal recurrence agree with the reports published, in that this type of recurrence usually occurs in patients in advanced stages with serosal involvement and nodal metastases [3-6, 8, 9, 23]. This female patient with a Krukenberg tumor underwent bilateral oophorectomy (Table 2), as recommended in the literature, resulting in an improved survival $[18,19]$.

Three of our five patients with resection for recurrence had no symptoms at the time recurrence was diagnosed, and diagnosis was based on different methods in each individual patient (markers, endoscopy, CT). This supports the reports by other authors of a low sensitivity of auxiliary methods for diagnosing recurrence, and the idea that no single method is sensitive enough to recommend its use as the only method for patient follow-up $[1,21]$. The two symptomatic patients had dysphagia as their common symptom. These data are similar to those reported by Takeyoshi et al. [16], in that we both found the same percentage of symptom-free patients, and the diagnosis of recurrence was suspected based on laboratory test results, with an increase in CA 19-9 levels in their patient.

While in our overall practice most recurrent gastric cancers occurred within 2 years, which agrees with reports by many other authors, in the present group of patients who underwent surgery for recurrence the diagnosis was made after that time in three patients, and more than 1 year after surgery in the remaining two patients [2-6, 9, 10, 21].

The 26-month survival achieved using surgery alone in our small series of patients with recurrent gastric cancer is somewhat higher than the survivals reported by Takeyoshi et al. [16] and Shchepotin et al. [21], who administered adjuvant therapy as well to most of their patients. Our only patient who died (the patient with a Krukenberg tumor) had a 13-month survival from the performance of adnexectomy, shorter than that reported by Cheong et al. [18] and Kim et al. [19].

In conclusion, although the number of patients treated in our series was low, we think that surgical treatment is a valid alternative in selected patients with recurrent gastric cancer, but this treatment should be performed by work teams specialized in this type of surgery. Surgical morbidity and mortality are low, and a longer survival may be achieved.

\section{References}

1. Bohner H, Zimmer Th, Hopfenmuller W, Berger G, Buhr H. Detection and prognosis of recurrent gastric cancer. Is routine follow-up after gastrectomy worthwhile? Hepatogastroenterology 2000;47:1489-94.

2. Bennett J, Gonen M, D'Angelica M, Jacques D, Brennan M, Coit D. Is detection of asymptomatic recurrence after curative resection associated with improved survival in patients with gastric cancer? J Am Coll Surg 2005;201:503-10.

3. Schwarz R, Zagala-Nevarez K. Recurrence patterns after radical gastrectomy for gastric cáncer: factors and implications for postoperative adjuvant therapy. Ann Surg Oncol 2002;9:394-400.

4. D'Angelica M, Gonen M, Brennan M, Turnbull A, Bains M, Karpeh S. Patterns of initial recurrence in completely resected gastric adenocarcinoma. Ann Surg 2004;240:808-16.

5. Yoo C, Noh S, Shin D, Choi S, Min J. Recurrence following curative resection from gastric carcinoma. Br J Surg 2000;87:236-42.

6. Roukos D, Kappas A. Limitations in controlling risk for recurrence after curative surgery for advanced gastric cancer are now well-explained by molecular-based mechanisms. Ann Surg Oncol 2001;8:620-1.

7. Kappas A, Roukos D. Quality of surgery determinant for the outcome of patient with gastric cancer. Ann Surg Oncol 2002;9: 828-30.

8. Maehara Y, Hasuda S, Koga T, Tokunaga E, Kakeji Y, Sugimachi K. Postoperative outcome and sites of recurrence in patients following curative resection of gastric cancer. Br J Surg 2000;87: 353-7.

9. Averbach A, Jacquet P. Strategies to decrease the incidence of intra-abdominal recurrence in resectable gastric cancer. Br J Surg 1996;83:726-33.

10. Marrelli D, Roviello F, de Manzoni G, Morgagni P, Di Leo A, Saragoni L, et al. Different patterns of recurrence in gastric cancer depending on Lauren's histological type: longitudinal study. World J Surg 2002;26:1160-5.

11. Lehnert T, Rudek B, Buhl K, Golling M. Surgical therapy for loco-regional recurrence and distant metastasis of gastric cancer. Eur J Surg Oncol 2002;28:455-61.

12. Fujii K, Fujioka S, Kato K, Machiki Y, Kutsuna Y, Ishikawa A, et al. Resection of liver metastasis from gastric adenocarcinoma. Hepatogastroenterology 2001;48:368-71.

13. Okano K, Maeba T, Ishimura K, Karasawa Y, Goda F Wakabayashi $\mathrm{H}$, et al. Hepatic resection for metastatic tumors from gastric cancer. Ann Surg 2002;235:86-91.

14. Ambiru S, Miyasaki M, Hiroshi I, Nakagawa K, Shimizu H, Yoshidome $\mathrm{H}$, et al. Benefit and limits of hepatic resection for gastric metastases. Am J Surg 2001;181:279-83.

15. Weitz J, Blumgart L, Fong Y, Jarnagin W, D'Angelica M, Harrison L, et al. Partial hepatectomy for metastases from noncolorectal nonneuroendocrine carcinoma. Ann Surg 2005;241: 269-76.

16. Takeyoshi I, Ohwada S, Ogawa T, Kawashima Y, Ohya T, Kawate $\mathrm{S}$, et al. The resection of non-hepatic intraabdominal recurrence of gastric cancer. Hepatogastroenterology 2000;47:1479-81.

17. McGill FM, Ritter DB, Rickard DB, Kaleya CS, Wadler RN, Greston WM, et al. Krukenberg tumors: can management be improved? Gynecol Obstet Invest 1999;48:61-5.

18. Cheong JH, Hyung WJ, Chen J, Kim J, Choi SH, Noh SH. Survival benefit of metastasectomy for Krukenberg tumors from gastric cancer. Gynecol Oncol 2004;94:477-82.

19. Kim H, Heo D, Bang Y, Kim N. Prognostic factors of Krukenberg's tumor. Gynecol Oncol 2001;82:105-9.

20. Wittekind $\mathrm{CH}$, Greene FL, Hutter R, Klimpfingerand M, Sobin LH (eds.). TNM atlas: Illustrated guide to the TNM classification of malignant tumours. 5th ed. Heldelberg: Springer Verlag; 2005.

21. Shchepotin I, Evans SR, Shabahang M, Cherny V, Buras RR, Zadorozhny A. Radical treatment of locally recurrent gastric cancer. Am Surg 1995;61:371-6.

22. Guadagni S, Catarci M, Kinoshita T, Valenti M, Bernardinis G, Carboni M. Causes of death and recurrence after surgery for early gastric cancer. World J Surg 1997;21:434-9.

23. Marrelli D, De Stefano A, de Manzoni G, Morgagni P, Di Leo A, Roviello F. Prediction of recurrence after radical surgery for gastric cáncer. A scoring system obtained from a prospective multicenter study. Ann Surg 2005;241:247-55. 